\title{
DIGITAL HUMANITIES AND STUDY OF LITERATURE: PERSPECTIVES IN DISTANCE EDUCATION
}

\author{
DR. SILIMA NANDA \\ Deputy Director, REC, IGNOU, Bhubaneswar, India
}

\begin{abstract}
In Distance Education reading, analyzing and interpreting learning material for students is an exhausting task because of the absence of the real teacher. In the 21st Century however, with the onset of digital revolution, things have drastically changed and now students feel comfortable accessing the texts through the tools of "digital humanities". This expression can be taken as a single word but it is a combination of two words 'digital 'and 'humanities', where the former refers to the technological component of the area of humanities and social sciences. Distance Education being global in character defies physical boundaries. Therefore, it is easy on the part of the students to experiment with the digital tools and understand the process of handling new technology on a collaborative platform. My article explores how to apply digital humanities specifically for the teaching of English and convert the electronic class into an attractive, dynamic and interactive platform.

KEYWORDS: Digital, Humanities, Distance Education \& Collaborative
\end{abstract}

Received: Jun 29, 2021; Accepted: Jul 19, 2021; Published: Jul 31, 2021; Paper Id: IJESRDEC20212

\section{INTRODUCTION}

\section{What is Digital Humanities?}

Digital Humanities is the intersection of new computational approaches to the traditional humanistic scholarship. In other words, Digital Humanities can be taken as an umbrella term for a number of activities that involves technology and human scholarship like visualization, digital repositories, data mining, cloud computing, etc. In this manner, learning becomes not only systematic and enriching; it also accelerates the pace of the learning process. The students with the help of visual tools like graphics, animation, images etc. represent their data. During the process of learning, students are free to access the online platform creating their own websites, editing information and creating individual portals. It is interesting to note the interdisciplinary nature of such a learning process, where students not only use the digital tools but also enjoy the freedom to modify and reconstruct the field of computing. In its initial stage, DH was used by a small group of scholars. Owing to its easy applicability; it spread to a large section of scholars who could access the system any time, $(24 \times 7)$. The infrastructure of $\mathrm{DH}$ is a dynamic one that arouses the enthusiasm of learners to understand the pedagogy and technology of the learning process.

\section{The Ideal Mode}

The learning materials of Distance Education are generally assimilated and drawn from different sources and comprehensively consolidated in form of Units and Blocks forming different Course Modules. The sources of information include compendium of texts, projects, primary and secondary sources. As the body of information is vast there is a need to systematize its structure and categorize the resources into different patterns. This is where the need for Digital Humanities arises. Digital Humanities is an ideal mode for collaborative, transdisciplinary and 
computationally engaged learning. It allows scholars to assess old problems with a new approach which otherwise would not have been possible in a conventional school of learning. Now the issue is that in such a novel set-up what precisely would be the role of the teacher? When information is immediately accessible to the students from their mechanical device, the learner is faced with the conflict of what to select and what to omit. Different paths of information place the learner in an ambiguous situation and this is where the teacher's presence is required to guide assist and filter the required information to the students. Like a facilitator, the teacher helps the students to assimilate new knowledge from multimodal sources of the digital platform. The teacher motivates the students to acquire, understand and build upon a new interpretation or knowledge of their own.

\section{Distance Learning and Digital Humanities}

Distance Learning is a flexible and informal platform, for students in this educational and professional field, Digital Humanities is the ideal solution as teaching and research, sharing of educational practices, innovations can create larger learning opportunities. Here the word 'Open' refers to the flexibility of interpretation, mixing and re-using of contents through open licenses so that the counselors or teachers don't have to make efforts to develop fresh educational materials; rather they can go for sharing. Mark Sample has aptly observed the "naked, increasing accountability and replicability" of Digital Humanities. The ideal factor in Digital Humanities is that it gives the opportunity to the students for editing, modifying and organizing their contents with the latitude to re-design them. All this together makes the learning process interactive and collaborative where the students can share their views with their peer groups on a common platform. Digital Humanities can encompass diverse curriculum like Geography, History, Sociology, ICT, Media Studies etc. The nature of subjects becomes interdisciplinary. During the course of learning if the students desire they can create their own folders, eportfolios from which they can access and store all information. Open networked education can solve the issues of students across the world in form of creating groups of students/ mentors and this approach can be made purely modular. Diverse skills of students can be generated like the honing skills for developing projects, technical skills for database development, and management skills for marketing and software skills to interact and communicate with the peer groups

\section{English Studies through Digital Tools}

Literature and linguistics have a close connection with the world of computers. It is said that composition and computers go together. Reading, analyzing and interpreting a literary work may be a time-consuming and exhausting task especially for those who are not bookworms. The teacher's role is to motivate students to read literary works and develop their critical thinking. In the age of digital natives where everything starts and ends with a 'click' on the keyboard, this seems to be a far-fetching undertaking. However, with the help of audio-visual devices and various online digital tools English literature might trigger motivation and encourage the learning process. It was during the 1980s that digitization of canonical texts took place, giving rise to experimental writing (hypertext fiction). Since the last decade onwards with the technological inventions of Ipads, kindles, tablets enormous projects of translations of literary works have taken place. This has enhanced the readership, reinforcing the close coordinates of digital technology, reading and textuality. Due to the multi-lingual translation of texts, there has been an upsurge of e-readers. Google books have become the buzzword in the global community. The audio texts with the interplay of music and background effects have captured the interest of the learners.

Thus a cultural revolution through Digital Humanities has beautifully transformed the theoretical world of literature into a magical, vibrant and immediate reality. The teaching of English literature is a challenging task as it crystallizes the attitude of the students to deal with Reality and imagination beyond the language boundaries. DH tools can be used in classrooms 
for understanding literary texts. In order to discuss Dickens's novels, the best way is to use the digital tool of video or animation which would enhance observation and critical thinking. Likewise, teaching Shakespeare's plays and sonnets to a group of young people can be intimidating as it is necessary to familiarize students with the era of Renaissance, its language, habits, circumstances, which are distant to the young people living in the age of the Internet, email, smartphones, and other educational digital devices. One of the most effective tools in bridging this gap is the medium of film. In this manner, the classical texts are brought into the postmodern sphere. Giving students collaborative learning assignments is also a fruitful teaching strategy as it facilitates students to work at solving multiple problems in groups. Also, using film adaptations of video games in teaching literature significantly improves students' achievements as students are both entertained and taught at the same time. In this manner the students' feelings are awakened, senses sharpened, and thoughts deepened. In order to interpret Wordsworth's poetry, the learners can virtually travel to the Lake District and using a map find out the geographical locations mentioned in his poems. In such a manner, history, geography, and culture are blended to have a real feel of the spirit of the text. Likewise to understand Sherlock Holmes students can connect the themes, characters and locations mentioned in the novel through a virtual map that makes the reading inter textual and interdisciplinary. The students learn to integrate the texts with the milieu of the specific period. Thus through the use of digital tools the teacher can goad the students to navigate beyond the boundaries of the physical classroom and explore the virtual world The new media studies is another area of humanities which has given birth to a novel digital culture comprising of blogs, video games, Twitter, Whatsapp, facebook, interactive maps, cartoon films et al. Students enjoy the freedom of not only viewing the blogs and wikis but also contribute further contents, podcasts to be shared online. With the decline of technical barriers students of literature find it easy and interesting to share their reading and develop projects over interactive digital devices like smartphones. Digital literacy thus helps in developing a critical disposition towards how to use a new piece of technology encompassing "an understanding of the role humans play in questioning, challenging and therefore shaping this techno-social system" [ Pangrazio 2016, 169]. It also helps in the development of research projects.

The role of the teacher is very significant for making the students understand and apply tools of Digital Humanities, to inculcate the art of critical thinking and develop analytical ability. For such students who do not have access to technology, the teacher has to create their digital awareness, clear the levels of difficulty in accessing, exploring and selecting the digital resources. Teachers can motivate the students to create their individual and online digital portals, develop skills to synthesize the digital resources in a collaborative manner. For sustaining such an intellectual effort, the teacher himself should be abreast with all the developments happening in the digital world.

\section{Data Visualization and Game Design}

Web-based tools are "both intuitive enough for lower-level undergraduates to learn without much coaching and powerful enough to show how computers have expanded the ways we can interact with texts and analyze their content" [Ficke 2014 ]. Teachers can resort to various data visualization tools and assist students to analyze the subjective representations of any literary piece say of poetry or drama. Using games to teach skills and concepts is an ancient concept, and even the use of video games for educational purposes is a concept that its origin in the 1970s (Epper, Derry berry, \& Jackson, 2012). Games can be a very rewarding method of instruction for learners and a good way of teaching soft skills. Games and gamification can be extremely engaging particularly if the game can facilitate a flow experience or a state of complete absorption or engagement in an activity. This will motivate the students to broaden their learning experiences using digital 
tools and strengthen their analytical skills. For example, the puritanical settlement in the New England of the US can be beautifully demonstrated through graphs, maps and the virtual routes of the early settlers instead of theoretically teaching the historical background. The role play of the students can be done through simulations or creating a game environment where students can directly partake, journeying along with the characters created by the author. Teachers can incorporate activities like meme creation, multimedia projects and other social media interventions which would help the learners to blend both the real and the virtual, past and the present and thus promote their digital perceptions.

In the digital space, the students learn to deconstruct stories, legends, myths and know how to reconstruct them into something new. Thus in a collaborative manner the new knowledge is distributed among the learners through social networking and wiki platforms. Teachers help students "to weave-to build, to fabricate, to design" and engage "with seemingly incongruous materials while developing a critical thinking practice about the process and the product" [Sample 2012]. Thus digital humanities promote public scholarship and help students to communicate in digital context.

\section{CONCLUSIONS}

Present day workplace requires that learners create and collaborate within the constraints of time and allow real-time communication among peers and co-workers who stay connected over the Internet. It is no coincidence that these advancements bring a fresh promise to distance education. Researchers and practitioners are recognizing emerging technologies as powerful tools for building social interaction in constructivist learning environments, assimilating the objective and the subjective worlds, reality and simulations, the past and the present in a single time and on a single platform. Thus the ever-evolving nature of technology will continue to push distance educators to use new digital tools to create learning environments that will prepare students to be life - long learners, who can problem solve through collaboration with global partners.

\section{REFERENCES}

1. Ficke, S. H. (2014). “From Text to Tags: The Digital Humanities in an Introductory Literature Course." The CEA Critic 76(2): 200-210.

2. Gavin, M., \& Smith, K. M. (2012). An Interview with Brett Bobley. In M. K. Gold (Ed.) Debates in the Digital Humanities, pp.61-66 University of Minnesota Press.)

3. Kirschenbaum, Matthew G. "What Is Digital Humanities and What's It Doing in English Departments?" ADE Bulletin 150 (2010): 55-61. Print.

4. Terras, M(2011). "Quantifying Digital Humanities." Retrieved from http://www.ucl.ac.uk/infostudies/melissaterras/DigitalHumanitiesInfographic.pdf

5. Pangrazio, L. (2016). “Reconceptualising Critical Digital Literacy.” Discourse: Studies in the Cultural Politics of Education 37 (2): $163-174$

6. Rockwell and Sinclair 2012 Rockwell, G., \& Sinclair, S. (2012). “Acculturation and the Digital Humanities Community.” In B. D. Hirsch (ed), Digital Humanities Pedagogy: Practices, Principles and Politics, Cambridge, UK: Open Book Publishers, pp. 177-211.

7. Sample, M. L. 2012. “What's Wrong with Writing Essays.” In M. K. Gold (ed), Debates in the Digital Humanities, University of Minnesota Press. Retrieved from http://dhdebates.gc.cuny.edu/debates/text/42 
8. Svensson, Patrik. "Humanities Computing as Digital Humanities.” Digital Humanities Quarterly 3.3 (2009): n. pag. Web. 2 Aug. 2010.

9. _ _ “The Landscape of Digital Humanities.” Digital Humanities Quarterly 4.1 (2010): n. pag. Web. 2 Aug. 2010. 
